\title{
Elastic waves in sintered packings of planar discs and rings
}

\author{
Jordan Jarrett $^{1}$ • Paul R. Heyliger ${ }^{1}$
}

Received: 22 January 2015 / Revised: 29 May 2015 / Accepted: 1 June 2015 / Published online: 9 June 2015

(C) OWZ 2015

\begin{abstract}
Plane elastic waves in assemblies of solid or hollow circular particles are considered using finite element approximations combined with periodic boundary conditions. Of primary interest are the dispersion relations and their interaction with particle geometry, the nature of the slowness curves for this class of particle assembly, the influence of particle anisotropy, and the effective elastic constants of these assemblies as computed by wave speed parameters. A comparison with experimental results from the literature is also provided for the case of extremely thin isotropic rings.
\end{abstract}

Keywords Wave Particle $\cdot$ Slowness $\cdot$ Finite-element . Periodic $\cdot$ Anisotropic

\section{Introduction}

Planar packings of discs are a class of varying-density medium that have often been represented, with varying degrees of accuracy, by effective continuum models. In both static and dynamic response of particle packings, measures such as effective bulk stiffness or wave speed are nearly always given as a function of the applied pressure. There have been several studies, both experimental and computational, focused on the dynamic response of particle packings. Experiments on assemblies of spheres performed by Duffy and Mindlin [1] indicated that the dependence on the effective elastic moduli of the aggregate material was of the form $\mu \tilde{p}^{\frac{1}{2}}$, where $\mu$ is a generic symbol for an elastic constant and $p$ represents the confining pressure. However, the Hertzian

Paul R. Heyliger

prh@engr.colostate.edu

1 Department of Civil and Environmental Engineering, Colorado State University, Fort Collins, CO 80523, USA contact model assumed in many theoretical studies indicates a power law of the form $\mu \tilde{p}^{\frac{1}{3}}$ [2-5].

There have been some modifications to the 3D Hertz model, including those of Goddard [6] and the study of a twodimensional hexagonal close-packed lattice of 3D elastic frictional balls by Velicky and Caroli [7]. This latter system was also studied by Coste and Gilles [8] in an effort to determine the limits of Hertzian contact for acoustic predictions. There have also been some simulations, notably by Melin [9], who found that for a gravity-packed two-dimensional lattice the speed of sound did not possess the standard power-law dependence. Jia et al. [10] performed experiments of granular glass beads and found that the effective sound velocity follows a quasi power law, where the exponent varies from a low value of approximately $1 / 4$ to the Mindlin-Hertz value of $1 / 6$. A number of other significant studies have presented excellent estimates on the nature of wave behavior in granular media using a variety of numerical and experimental methods [11-16].

There is a significant change in behavior of granular materials when the contact zone between particles changes to one of bonding [17] or cementing [18]. This causes an increase in contact stiffness that has been shown to change the elastic properties of the bulk medium [19] and potential damage mechanisms [20].

In this study, the idealized response of square packings under plane elasticity conditions are studied to determine the bulk dynamic behavior of these structures when excited by planar waves. The geometries considered here are a departure from many existing studies in that there is no applied confining pressure. Instead, displacement and stress continuity are assumed at the contact points between particles based on their initial configuration prior to the application of any such pressure. The particle contact points are sintered in the sense that the continuity conditions at these locations are assumed 
to be maintained during the course of the deformation. The periodic nature of these configurations allows simplification in meshing to assess the frequency behavior as the particle changes from being fully solid to approaching the geometry of a hollow ring. The constitutive properties of the individual particles can be either isotropic or anisotropic, but in this study they are limited to orthotropic solids whose primary material axes align with the bounding walls of the packing.

\section{Theory}

\subsection{The general domain}

A collection of circular elastic discs is considered with uniform outer radius denoted by the lowercase letter $a$ and potential inner radius denoted by the letter $R$. Each disc is composed of material that can be considered linearly elastic and is represented by the contracted elasticity tensor components $C_{i j}$, the area $A$, and the bounding curve $C$. The surface region of each particle, represented by the circumference of the circular boundary, touches four other particles at $90^{\circ}$ intervals on $C$. It is assumed that each of these particle contacts is in perfect sticking contact. It is also assumed that displacements and tractions are continuous at these contact locations and remain so during the deformation. This is a significant restriction, as it implies that the displacements between two connecting particles at the contact points are identical and the forces between particle contacts are equal and opposite. This is an idealized behavior that is assumed to hold so that limits on the dynamic characteristics of the entire packing can be inferred. The deformation is assumed to be sufficiently small that the equations of linear smallstrain elasticity hold, and that no further particle contacts are formed during the deformation. There is no assumed force-displacement relation other than that obtained using the equations of motion and boundary/interface conditions as described later in this section.

\subsection{Governing equations}

The in-plane equations of motion for a planar solid under either plane stress or plane strain conditions are given in rectangular Cartesian coordinates as

$$
\begin{aligned}
& \frac{\partial}{\partial x}\left[C_{11} \frac{\partial u}{\partial x}+C_{12} \frac{\partial v}{\partial y}+C_{16}\left(\frac{\partial u}{\partial y}+\frac{\partial v}{\partial x}\right)\right] \\
& +\frac{\partial}{\partial y}\left[C_{16} \frac{\partial u}{\partial x}+C_{26} \frac{\partial v}{\partial y}+C_{66}\left(\frac{\partial u}{\partial y}+\frac{\partial v}{\partial x}\right)\right] \\
& +f_{x}=\rho \frac{\partial^{2} u}{\partial t^{2}} .
\end{aligned}
$$

$$
\begin{aligned}
& \frac{\partial}{\partial x}\left[C_{16} \frac{\partial u}{\partial x}+C_{26} \frac{\partial v}{\partial y}+C_{66}\left(\frac{\partial u}{\partial y}+\frac{\partial v}{\partial x}\right)\right] \\
& +\frac{\partial}{\partial x}\left[C_{12} \frac{\partial u}{\partial x}+C_{22} \frac{\partial v}{\partial y}+C_{26}\left(\frac{\partial u}{\partial y}+\frac{\partial v}{\partial x}\right)\right] \\
& +f_{y}=\rho \frac{\partial^{2} v}{\partial t^{2}}
\end{aligned}
$$

Here $u$ and $v$ are the displacement components in the $x$ and $y$ directions, respectively, $\rho$ is the material density, $f_{x}$ and $f_{y}$ are the $x$ and $y$ components of the body force vector, and $t$ is time. The $x$ and $y$ components of the body force vector are frequently assumed to be sufficiently small and are therefore neglected. Although the nature of the circular particles makes the choice of cylindrical coordinates a potential alternative, the imposition of periodic boundary conditions over representative subdomains of the particle packings make rectangular Cartesian coordinates a more robust choice for any general packing.

The choice of using a full elasticity tensor to represent the relationship between effective force and displacement within the particle is one of several methods used to link these variables and has been used in the past to study a variety of problems in particle mechanics [21]. It is also possible and fairly well established that simplified one-dimensional models can be used to represent this sort of behavior [22-24].

The weak form of these two equations can be obtained by multiplying each of the equations of motion by an arbitrary weight function, integrating over the domain of a typical finite element $\Omega_{e}$, integrating by parts, and combining the result with the assumption that the temporal response is of the form $\mathrm{e}^{j \omega t}$, where $j=\sqrt{-1}$ and $\omega$ is the wave frequency. This yields a linear system in terms of nodal values of $u$ and $v$ for either the element or the entire system that can be expressed in matrix form as

$[K]\{U\}-\omega^{2}[M]\{U\}=\{F\}$.

These matrices are quite standard and details of their composition are not included here. In all cases that follow, four-node quadrilateral elements with bilinear approximations of each displacement component were used along with full numerical integration of all matrix entries. The usual steps of element assembly, imposition of boundary conditions, and solution of the primary variables proceeds in typical fashion [25] other than the consideration of periodic boundary conditions as described below.

\subsection{The square packing}

The instantaneous stiffness of any particle packing depends primarily on the original particle configurations and interactions with neighbors, along with the components of the elastic stiffness tensor of individual particles. The main intent here 


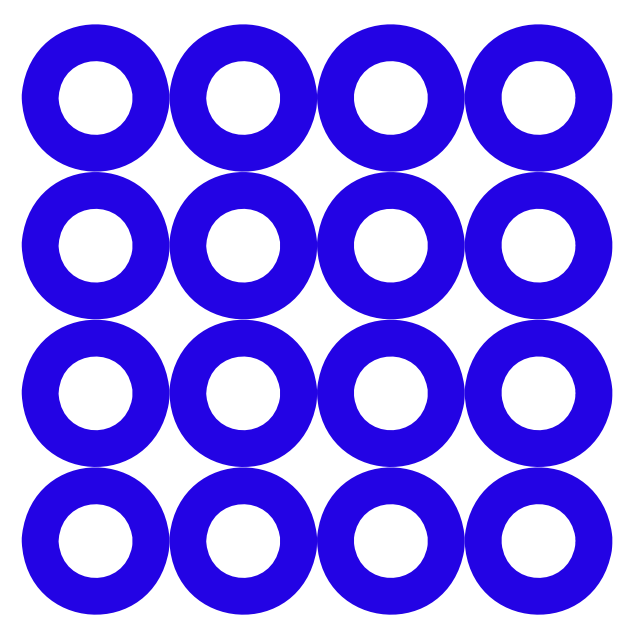

Fig. 1 An array of circular rings arranged in a square packing

is to explore the influence of aggregate structure and localized variance in particle elasticity on the transient behavior of square particle packings with perfect bonding assumed between particle contacts. What follows in this work is a significant idealization of true particle behavior during transient motion. It is assumed that the change in contact length because of compressive force, or the loss of contact between two particles, can be neglected for this initial analysis so that basic wave behavior can be determined by examining the bulk behavior of the particles. The results that follow should be viewed with this restriction in mind.

Square packings, such as that shown in Fig. 1 for a representative subdomain of rings, are simple in structure. They have four points of contact between particles and maintain these initial contact points during the course of any deformation. They also do not possess the properties that are characteristic of random packings, such as having numerous potential contacts that can be realized as the confining pressure increases. Yet some information can be gained about the aggregate behavior of these collective particle interactions as their bulk properties are sought when the particles are subject to a planar incident wave. Square packings were considered primarily because their periodic boundary conditions are straightforward to visualize and implement and also because the finite square ring packing is one of the few configurations for which there are experimental results.

\subsection{Periodic boundary conditions}

Ordered packings possess two huge advantages over more generic packings: their consistency in number and location of contact points and the periodic nature of their microstructure. These two features can be exploited to greatly reduce the domain of the particle packing to the level of just a few elements or, in the case of the square packing, a single particle.
The packings considered in these analyses are assumed to be infinite in both $x$ and $y$ directions and maintain their periodic structure in these directions. The entire structure is excited by a plane wave characterized by the wave vector $\mathbf{k}$ with magnitude $k$. This wave acts at an angle $\theta$ measured clockwise from the positive $y$-axis. The time dependence is the same as that denoted in the earlier section.

Unbounded particle packings can be doubly periodic, and hence each of the two displacement components (along with the strains and stresses) must each satisfy the Bloch condition [26]. For any elastic field variable $G$, this can be expressed as

$G\left(x+d_{x}, y+d_{y}\right)=e^{j \phi_{x}} e^{j \phi_{y}} G(x, y)$.

Here $\phi_{x}=d_{x} k \sin \theta$ and $\phi_{y}=d_{y} k \cos \theta$ where $d_{x}$ and $d_{y}$ represent the periodic distances in the $x$ and $y$ directions. In the case of the disc/ring these distances correspond to the outer diameter of the particle, or $2 a$. This condition allows the discretization of a single unit cell of the disc packing rather than a larger portion of the structure as the periodic nature of the cell edges can be used to reduce the equations of motion to consideration of a reduced eigenvalue problem of the condensed unit cell [27,28].

The case of the square packing is used to demonstrate this reduction. The packing shown in Fig. 1 has particles with contact points at $0^{\circ}, 90^{\circ}, 180^{\circ}$, and $270^{\circ}$ as measured from the positive $x$-axis. The lines of periodicity run tangent to these contact points and reduce the unit cell to a single disc, with these respective boundary nodes referred to as specific regions and labeled as $1\left(180^{\circ}\right), 2\left(270^{\circ}\right), 4\left(0^{\circ}\right)$, and $5\left(90^{\circ}\right)$. The remainder of the particle not coincident with a contact node is denoted as region 3 .

Two sets of boundary conditions are periodic in this case: the displacements at node (or region) 4 are periodic in $x$ with those of region 1 , and the displacements of region 5 are periodic with those of region 2 . These are expressed as

$$
\left\{U_{4}\right\}=\left\{U_{1}\right\} e^{j \phi_{x}}
$$

and

$$
\left\{U_{5}\right\}=\left\{U_{2}\right\} e^{j \phi_{y}} .
$$

There are similar relations for the forces at these locations, where the symbol $F$ could be used to replace the generic displacement $U$ in the $x$ and $y$ directions. Using these expressions, the total displacement vector for all nodal locations in the domain can be defined as $\{U\}$ and can be expressed in terms of only the particle displacements at the left boundary $\left\{U_{1}\right\}$, the lower boundary $\left\{U_{2}\right\}$, and the displacements in the interior of the particle $\left\{U_{3}\right\}$, using a simple transformation [28]. 


$$
\begin{aligned}
\{U\} & =\left\{\begin{array}{l}
\left\{U_{1}\right\} \\
\left\{U_{2}\right\} \\
\left\{U_{3}\right\} \\
\left\{U_{4}\right\} \\
\left\{U_{5}\right\}
\end{array}\right\}=\left[\begin{array}{rr}
{[I]} & {[0][0]} \\
{[0]} & {[I][0]} \\
{[0]} & {[0][I]} \\
e^{j \phi_{x}}[I] & {[0][0]} \\
{[0] e^{j \phi_{y}}[I][0]}
\end{array}\right]\left\{\begin{array}{l}
\left\{U_{1}\right\} \\
\left\{U_{2}\right\} \\
\left\{U_{3}\right\}
\end{array}\right\} \\
& =[P]\left\{U_{R}\right\} .
\end{aligned}
$$

Here $[\mathrm{I}]$ is the identity matrix. A similar relation can be derived for the forces at these locations, and is given as

$$
\{F\}=[P]\left\{\begin{array}{l}
\left\{F_{1}\right\} \\
\left\{F_{2}\right\} \\
\left\{F_{3}\right\}
\end{array}\right\} .
$$

Premultiplying the original finite element matrix equation by the conjugate transpose of the transformation matrix gives the final matrix expression as

$$
\begin{aligned}
& {[P]^{* T}\left([K]-\omega^{2}[M]\right)[P]\left\{U_{R}\right\}} \\
& \quad=\left(\left[K_{R}\right]-\omega^{2}\left[M_{R}\right]\right)\left\{U_{R}\right\}=[P]^{* T}[P]\left\{F_{R}\right\} .
\end{aligned}
$$

Here the $[K]$ and $[M]$ matrices are the original matrices that result from the finite element discretization in Eq. (3) and the subscript $\mathrm{R}$ refers to the reduced domain of the problem that contains only nodes corresponding to regions 1,2 , and 3. The critical matrices are defined as

$$
\left[K_{R}\right]=[P]^{* T}[K][P]
$$

and

$$
\left[M_{R}\right]=[P]^{* T}[M][P] .
$$

Both of the matrices have a similar form except that there are no inertial interactions between the original submatrices of the mass. The final form of the reduced stiffness matrix appears as

$$
\left[K_{R}\right]=\left[\begin{array}{ccc}
K_{11}+K_{44} & 0 & K_{13}+K_{34} e^{-j \phi_{x}} \\
0 & K_{22}+K_{55} & K_{23}+K_{35} e^{-j \phi_{y}} \\
K_{13}+K_{34} e^{j \phi_{x}} & K_{35} e^{j \phi_{y}} & K_{33}
\end{array}\right] .
$$

Following formation of these equations, the frequencies and displacement patterns can be computed for a variety of conditions using a standard eigensolver.

\section{Numerical results}

After the periodic solid has been approximated using a collection of elements, the usual remaining inputs are the material properties and the wave vector information. The resulting outputs are the frequencies and the modal displacement vector $\{U\}$. In all results that follow, the components of the body force vector are assumed to be zero.

\subsection{Continuous media}

It is an instructive and useful exercise to apply the methodology described above to the case of an infinite medium. This allows the direct comparison of dispersion characteristics while simultaneously providing a sequence of comparison points for the behaviors of continuous media versus discontinuous particle packings.

Plane harmonic waves through an infinite planar homogeneous isotropic solid under plane strain conditions are considered first. The modeled domain is completely filled in this case and is of dimensions $2 d \times 2 d$. This is a very wellknown problem [28] whose exact solution for frequency in terms of the wave speed $c$, side length $2 d$ of an arbitrary square unit cell, and the $y$ component of the vector $\mathbf{k}$ can be expressed for a wave vector in the $y$ direction as

$\omega=c \sqrt{(n \pi / d)^{2}+\left(k_{y}+m \pi / d\right)^{2}}$.

Here the wave speed $c$ can take the value for either the shear wave speed of $\sqrt{C_{44} / \rho}$ or the longitudinal wave speed $\sqrt{C_{11} / \rho}$.

Three meshes were considered to represent this material: (1) a 4 x 4 mesh of linear elements (25 nodes), (2) a mesh of 280 nodes, and (3) a mesh of 1185 nodes and 1200 elements. The latter two meshes are shown in Fig. 2. They are hardly ideal for the infinite sheet, but are representative of the type of mesh that will be used for the particle packings in later studies and hence are good candidates for comparison here. In this case the periodicity exists along the $x$ and $y$ borders as well as the corners of each mesh, and the resulting element matrices must be adjusted accordingly.

The reduced frequency $\bar{\omega}$ is defined to be

$\bar{\omega}=\frac{2 d \omega}{\sqrt{\frac{E}{3(1-2 v) \rho}} .}$

Here $E$ is the modulus of elasticity and $v$ is the Poisson ratio for the isotropic material. The wave vector $\mathbf{k}$ is taken to be parallel to the $y$-axis for all results reported here. This restriction corresponds to a normal incident wave vector with $\theta=0$. For the results in this section, the parameters $d, C_{44}$, and $\rho$ were taken as unity and the Poisson ratio $v=0.325$. The remaining elastic constants can be computed from $C_{44}$ and $v$.

Very good estimates for the frequencies can be obtained even for relatively coarse finite element discretizations. In 

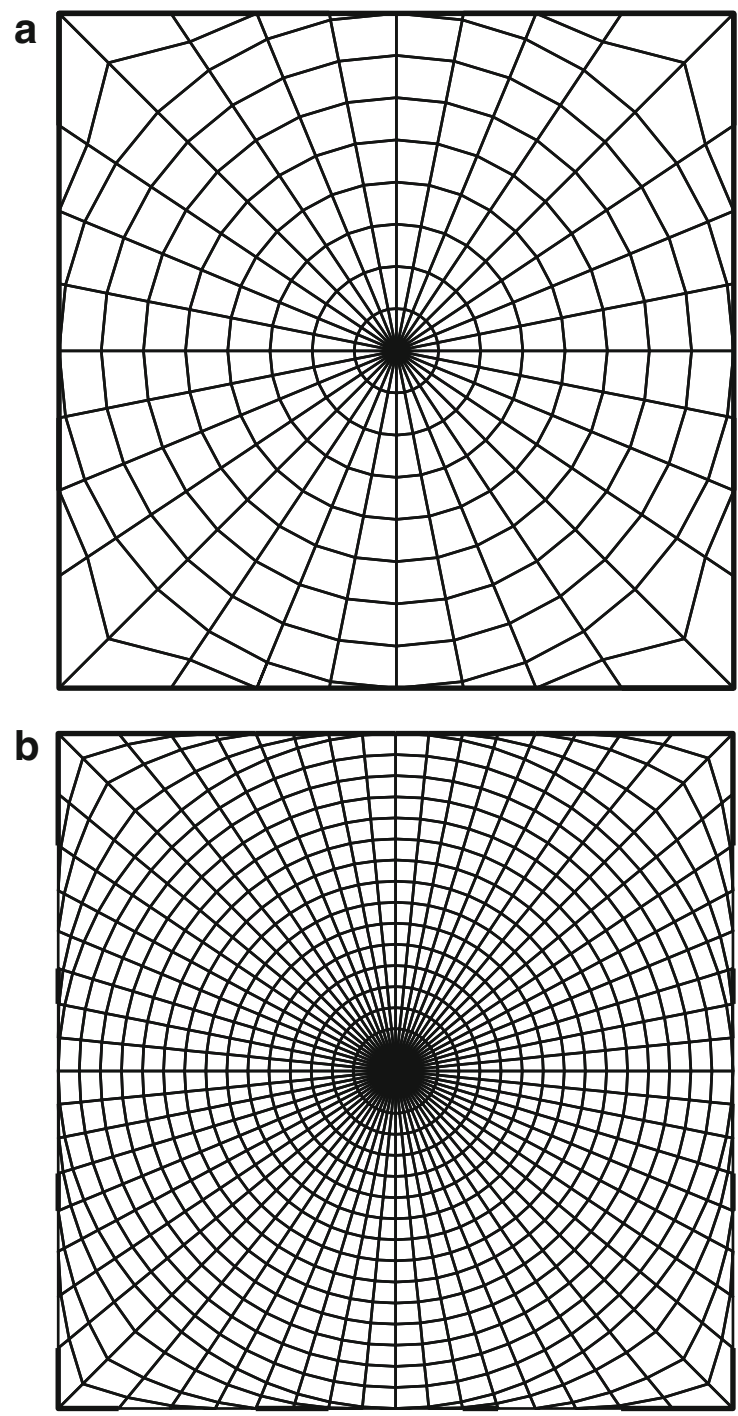

Fig. 2 The two meshes used to model a single circular disc

Table 1 Convergence of frequencies for solid continuum

\begin{tabular}{llllll}
\hline $\mathrm{m}$ & $\mathrm{n}$ & Mesh 1 & Mesh 2 & Mesh 3 & Exact \\
\hline 0 & 0 & 2.0286 & 1.9825 & 1.9787 & 1.9775 \\
0 & 0 & 3.9840 & 3.8892 & 3.8850 & 3.8837 \\
0 & 1 & 5.106 & 4.4723 & 4.4334 & 4.4219 \\
1 & 0 & 7.087 & 6.0656 & 5.9681 & 5.9326 \\
1 & 1 & 9.289 & 7.3518 & 7.1875 & 7.1301 \\
0 & 2 & 9.588 & 8.4314 & 8.2176 & 8.1535 \\
\hline
\end{tabular}

Table 1, the lowest six frequencies are given for the dimensionless frequency $\bar{\omega}$ as a function of the dimensionless wave number $\bar{k}=2 d k / \pi$. The more general dispersion behavior is shown in Fig. 3. The results are given in terms of the indicial integers $n$ and $m$, where the type of deformation for each mode indicated by combinations of $n$ and $m$ are given

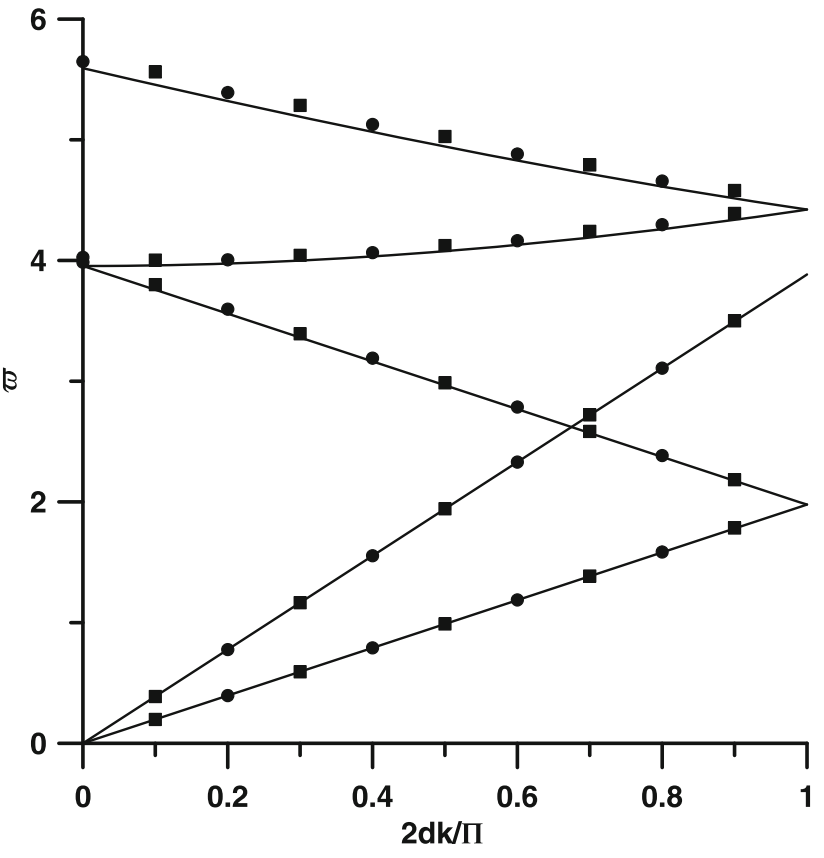

Fig. 3 Exact dispersion relations for a solid planar continuum (solid lines) compared with the finite element model for mesh 2 (square symbols) and mesh 3 (circular symbols). The character of the exact frequencies can be ordered from lowest to highest values of the exact curves at $2 d k / \pi=0.5$ by defining the values of $(n, m)$ in the analytic solution and if the mode is shearing or longitudinal as follows: $(0,0)$ shear, $(0,0)$ longitudinal, $(-1,0)$ shear, $(0,1)$ shear, and $(-1,1)$ shear

in Fig. 3. The coarse mesh provides estimates of the lower shear and longitudinal frequencies that are within 2-5\% of the exact values, with the higher modes at less accurate values that are over $10 \%$ in error. The second mesh has values that are far more accurate, with the lower six modes within several percent and the lowest shear and longitudinal modes within a fraction of a percent. This accuracy is even better for the final mesh, with the worst agreement being $3.4 \%$ for the sixth computed mode but the lowest shear and longitudinal frequencies off by 0.06 and $0.03 \%$ compared to the exact value. These results indicate that a mesh with the level of refinement used here is adequate to obtain sufficiently accurate results for the particle packings that follow.

\subsection{Square packings of isotropic discs and rings}

An array of square-packed circular discs can be modeled using a single disc along with periodic boundary conditions as described above with $d=2 a$ or the outer diameter of the representative disc. Unless otherwise noted we use uniform discs under plane strain conditions and study the dispersion relations for a variety of conditions. In all cases, we anticipate that the results from lower modes are well within $1 \%$ error based on the results for the infinite sheet in the earlier section. 


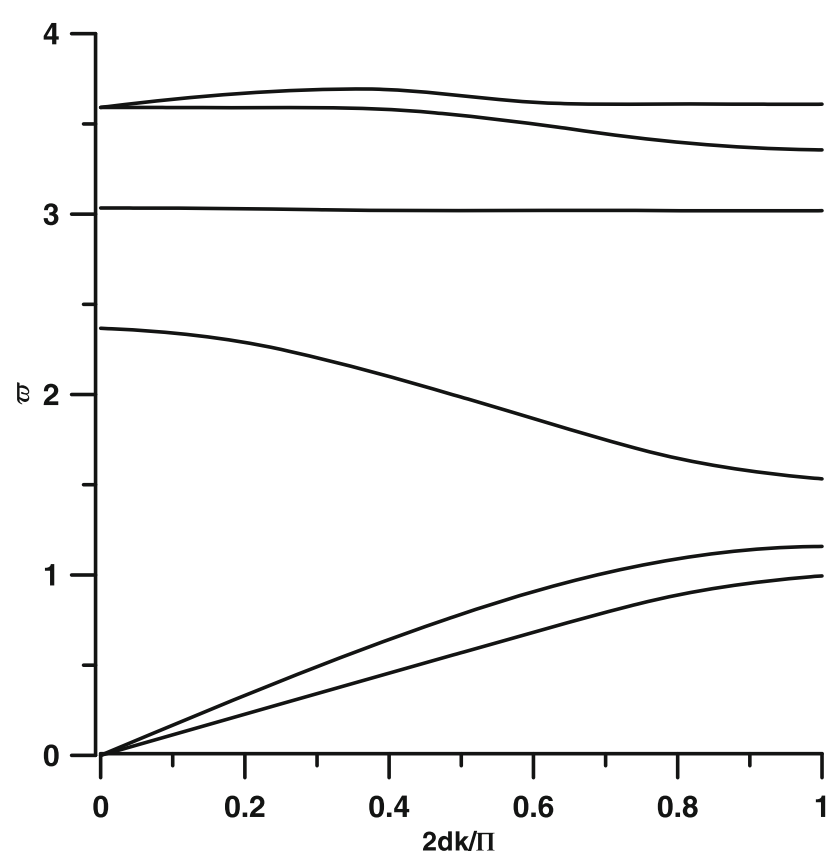

Fig. 4 The reduced frequencies of the lowest six modes for a square packing of solid isotropic particles as a function of $\bar{k}$

The primary assumption made regarding the kinematics of the particle packing is one of displacement continuity. Specifically, because of the imposition of periodic boundary conditions, the displacements at the points of contact are assumed to be continuous from particle to particle during the ensuing motion. Particle slip, rigid body rotations, or gaps between initial contacts are not represented during the class of deformation assumed in this model, but would of course be possible under specific conditions.

Of initial interest is a standard square packing of solid particles. Each particle is assumed to possess linear elastic behavior, and have a constitutive response that is represented by the modulus of elasticity $E$ and the Poisson ratio $v=0.3$. This problem is similar in nature to that of the previous section, except that rather than traveling through a continuous medium, the wave moves through a collection of particles over which much of the surface is traction-free. Because of this change in effective material density (from $100 \%$ fully filled to $78.54 \%$ ) and the consequent loss in stiffness, the issue is the change in the dispersion relations for the packing compared to a fully occupying solid. As in the previous section, unit values were used for $d, C_{44}$ and $\rho$.

The resulting dispersion curves for the square packing of solid particles are shown in Fig. 4, where the angular frequencies are shown as a function of the reduced wave number $\bar{k}$. These results were determined using a mesh that matches the circular domain of the mesh shown in Fig. 2 for the solid geometry but is now surrounded by a low-modulus material that effectively represents the traction-free condition of a void outside the particle boundary. This is a common method used to model voids in finite element simulations, and in this case the void modulus was eight orders of magnitude smaller than the modulus of the particle. Simulations were also completed for more coarse mesh simulations with the material entirely removed, and no differences were observed in the results. The resulting curves can be compared with the fully equivalent solid results from Fig. 3 and show the reduction in frequency for each respective case when the solid is replaced by the particle packing. The modal displacements for the lowest four modes corresponding to the lowest four curves in Fig. 4 are shown for a small $4 \times 4$ particle sub-array of the larger infinite domain of particles in Fig. 5a-d. The lowest mode maintains its shearing character, and the second mode shows the $y$ direction longitudinal behavior as the particle halves are compressed and extended. In terms of frequency, there is far less difference in value between the lowest shear and longitudinal modes than was the case for the continuum.

A related problem to the square packing of solid particles considers the question of how the wave propagation characteristics change as the mass of the particle changes by altering the amount of material within the particle. This is accomplished by considering an assembly of rings that have the same diameter (and hence contact points) as the solid particles but now with an inner radius of $R$ where $0<R<a$.

The dispersion curves for rings $(v=0.3)$ with varying thickness are shown for the lowest six modes in Fig. 6. Rather than changing the wave number, this value is fixed at $\bar{k}=1$ and the resulting reduced frequencies $\bar{\omega}$ are plotted for rings whose thickness varies from 0 for the solid particle $(R / a=$ $0)$ to a ring whose thickness is $1 / 16$ of the radius $(R / a=$ $15 / 16)$. These frequencies are plotted as individual points rather than curves since there is apparent modal crossing as the thickness of the ring decreases. In general, although the frequencies decrease along with wall thickness, this decrease is not proportional. The solid particle has the first six modes whose frequencies vary from approximately $1-4$, but for the ring with outer radius of 1 and a wall thickness of $1 / 16$, the lowest six frequencies vary from 0 to 1 . Hence for the thinnest ring considered, the frequencies are roughly $20 \%$ of their full-particle value while they are only $12 \%$ of their original volume. As is not uncommon for low-density media, ring packings appear to maintain a disproportionate level of stiffness as they are reduced in size for this specific geometry.

\subsection{Slowness curves and effective stiffness: isotropic and orthotropic particles}

Slowness curves can be considered for particle packings to assess the level of anisotropy in the bulk medium if the packing is treated as an effective continuum. The inverse of the quasi-transverse and quasi-longitudinal velocities are determined in the large wavelength limit for varying values of 


\section{a}

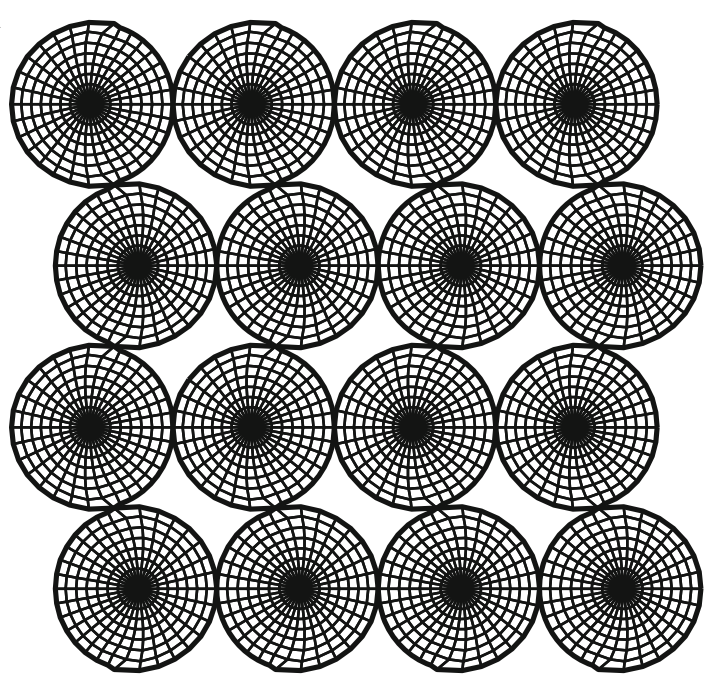

C

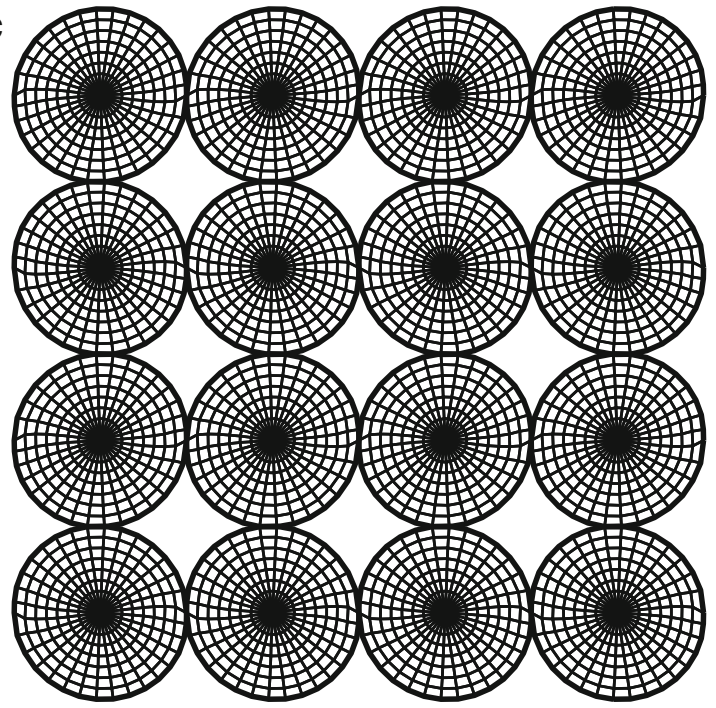

b

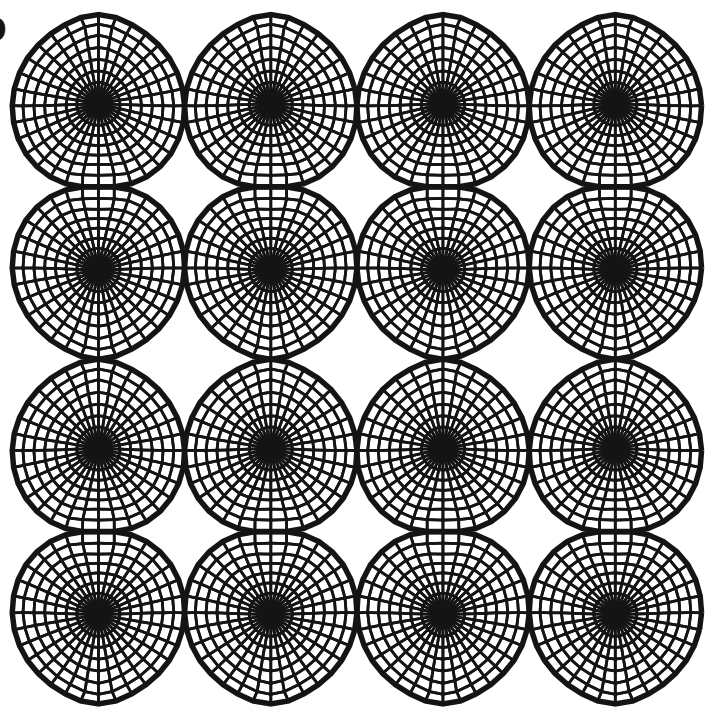

d

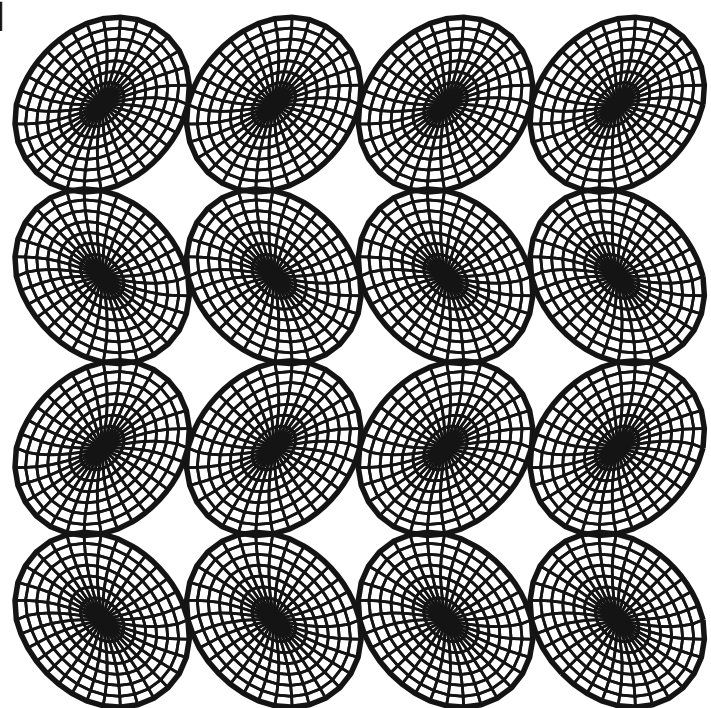

Fig. 5 a Mode 1, b Mode 2, c Mode 3, d Mode 4

the incident angle $\theta$. The limiting values are found by taking the linear low frequency extension of $\omega$ from the lowest two dispersion curves (the lowest is the transverse wave, the second is the longitudinal as shown in the modal plots for the isotropic packing). The wave speeds can then be computed using the relation

$c=\frac{\omega}{k}$.

Here $c$ is either $c_{T}$ for the transverse velocity or $c_{L}$ for the longitudinal velocity. These speeds can be computed for any incident wave angle $\theta$ and their inverse plotted against that angle. The same material properties were used for this analysis that were used in the previous section.

The slowness curves for rings with $a / R=0,0.25,0.5$, and 0.75 are shown in Fig. $7 \mathrm{a}-\mathrm{d}$. In each of these plots, the inverse of the effective shear wave speed are shown as solid lines and the inverse of the longitudinal wave speed are dashed. Initially, these curves are nearly circular for the fully filled particles, indicating behavior that is close to mimicing an isotropic solid. But as the discs become more and more hollow, there is a larger mismatch in the two curves and the lobes along the poles become more extended, giving effective behavior that is more anisotropic.

Since the velocities $c_{T}$ and $c_{L}$ generally vary with incident angle, the overall response of the square packing of rings can be viewed as an effective anisotropic medium with elastic constants that depend on but are not equal to the elastic constants of the individual particles. This material has an effective density of

$\tilde{\rho}=\rho \frac{\pi}{4}\left(a^{2}-R^{2}\right)$. 


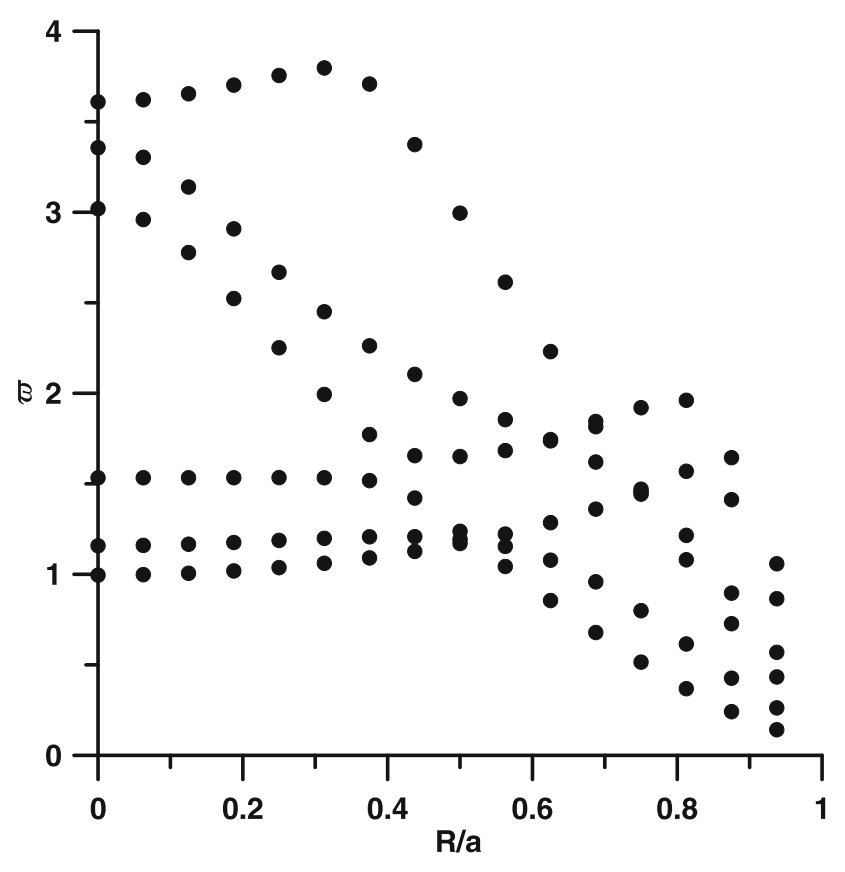

Fig. 6 The lowest six reduced frequencies for square packings of isotropic rings with varying thickness with $\bar{k}=1$. The points corresponding to $R / a=0$ are those of a solid particle

The material also possesses the elements of a two-dimensional effective elasticity tensor that can be given as

$[\Sigma]=\left[\begin{array}{ccc}\Sigma_{11} & \Sigma_{12} & 0 \\ \Sigma_{12} & \Sigma_{22} & 0 \\ 0 & 0 & \Sigma_{66}\end{array}\right]$

The indicial form of the Christoffel equation when the components of the elastic stiffness tensor for each particle are expressed in the usual four subscripts can be written as [26]

$$
\left(C_{i j k l} d_{j} d_{l}-c^{2} \rho \delta_{i k}\right) p_{k}=0
$$

Here $p_{k}$ are the components of the polarization unit vector, which represent the direction cosines of the particle displacement at any location in the ring. Purely longitudinal waves are generated when the polarization vector is parallel to the propagation direction $\mathbf{d}$, and pure shear waves are generated when the polarization vector is normal to the propagation direction. For the case of the particle packing, however, the element of the effective component of the elasticity tensor $\Sigma_{i j k l}$ can be extracted using the typical contracted notation where the four subscripts are reduced to only two [26]. For example, in the case of a wave propagating at $0^{\circ}$ and motion in the same direction, the reduction is enforced when the four subscripts are $\mathrm{i}=\mathrm{j}=\mathrm{k}=1=1$. In contracted notation, this implies

$\Sigma_{11}=\tilde{\rho} \tilde{C}_{L}^{2}\left(0^{\circ}\right)$
The tilde on the wave speed indicates the effective wave speed for the particle packing. Similar relations can be applied to give the two other effective stiffness coefficients as

$\Sigma_{66}=\tilde{\rho} \tilde{C}_{T}^{2}\left(0^{\circ}\right)$

and

$\Sigma_{12}=\tilde{\rho}\left(\tilde{C}_{L}^{2}\left(0^{\circ}\right)-2 \tilde{C}_{T}^{2}\left(45^{\circ}\right)\right)$.

Using this formulation, where the symbol $\Sigma$ represents the effective stiffness of the entire packing rather than the $C_{i j}$ stiffness elements of the individual particles, the effective cubic elastic constants $\Sigma_{11}, \Sigma_{66}$, and $\Sigma_{12}$ can be computed for the square packing for a variety of ring wall thicknesses. The variation of these effective constants relative to the individual particle modulus $C_{66}$ and are shown in Fig. 8 with $\Sigma_{11}$ (solid), $\Sigma_{66}$ (long dash), and $\Sigma_{12}$ (short dash) shown as a function of $R / a$. The effective elastic constants are roughly $1 / 4$ or $1 / 3$ of the particle elastic constants for the solid particle. The stiffness $\Sigma_{11}$ has an immediate decline as the particle becomes hollow while the shear modulus $\Sigma_{66}$ remains somewhat constant until $R / a$ reaches about 0.5 before starting to decline. The value of $\Sigma_{12}$ has more unusual behavior as it first increases then drops as $R / a$ exceeds 0.5 .

To assess the influence of the anisotropy of individual elements, a square packing of solid orthotropic particles is finally considered. The in-plane elastic constants are given as $E_{1}=155.0 \mathrm{GPa}, E_{2}=12.10 \mathrm{GPa}, v_{12}=0.248$, and $G_{12}=4.40 \mathrm{GPa}$ [29] with a density of $\rho=1800 \mathrm{~km} / \mathrm{m}^{3}$. The properties simulate those of a typical graphite-polymer composite, and of significant note is the large mismatch between the elastic modulus and the in-plane shear modulus. The particles have their stiff principal material direction located along the $x$-axis and the same analysis is completed as for the isotropic particle. The slowness curves for this packing are shown in Fig. 9. For this packing, the levels of anisotropy are now much more skewed toward the stiffer material axis of the particle. Although both the isotropic and orthotropic particles generate slowness curves that possess anisotropy of the quasi-transverse wave speeds, there is anisotropy in the quasi-longitudinal behavior for both types of particles. The nature of these curves is strongly similar to slowness curves obtained analytically for uni-directional graphite-epoxy composites [30] only with the reduced values consistent with the change from solid continuum to a packing of particles.

\subsection{Comparison with experiment}

Shim and co-workers [31] have performed experiments on several types of packing arrangements including the square- 

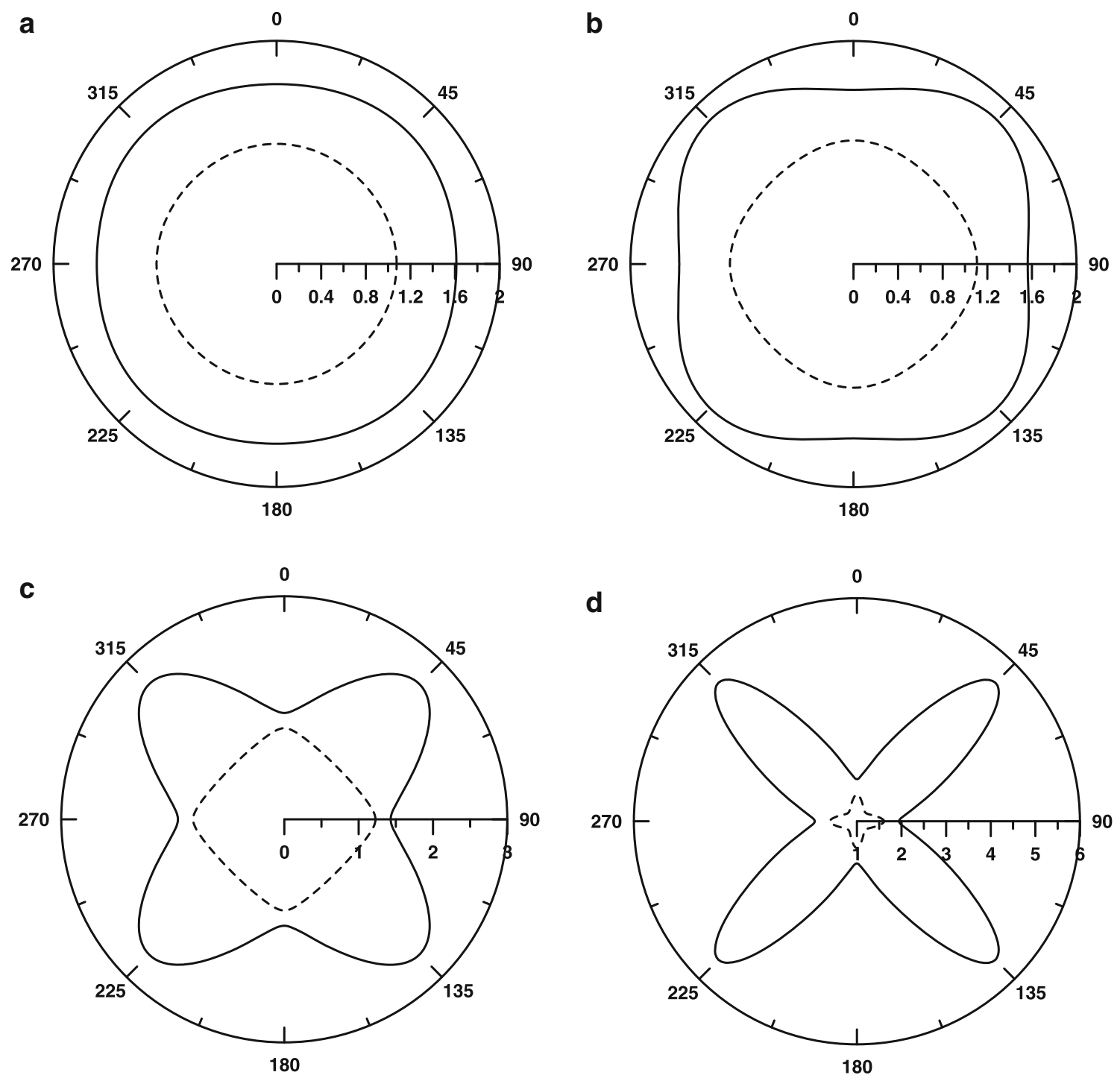

Fig. 7 a Slowness curves for the solid particle. b Slowness curves for the hollow particle $a / R=0.25$. c Slowness curves for the hollow particle $a / R=0.50$. d Slowness curves for the hollow particle $a / R=0.75$

packed array of rings considered in this study. They measured wave speeds for several different materials and ring geometries. The primary difference between their experimental arrangement and the idealized periodic structure considered here is the necessarily finite-sized array used in their experimental configuration. However, despite this limitation, their results provide one of the few studies of its type for this class of particle arrangement.

Two types of materials were studied. The first was brass, with a density of $8199 \mathrm{~kg} / \mathrm{m}^{3}$ and an elastic modulus of 120.4 GPa. The second was an aluminum alloy, with a density of $2693 \mathrm{~kg} / \mathrm{m}^{3}$ and an elastic modulus of $65.9 \mathrm{GPa}$. The values for Poisson ratio were not reported by Shim an co-workers, and values of 0.331 and and 0.334 were used for the brass and aluminum, respectively. The rings selected for comparison with the present study had a diameter of $38.1 \mathrm{~mm}$ and a wall thickness of $1 \mathrm{~mm}$. They were $10 \mathrm{~mm}$ in axial length.

The periodic domain used in prior sections require modification because of the nature of the experimental packings is no longer the same. Shim and co-workers [31] used the square-packed assembly shown in Fig. 10a. This packing is periodic only in the $y$ direction, and has a representative periodic domain shown in Fig. 10b. Only this latter section was modeled and only the $y$ periodic boundary conditions along the bounding tangents were imposed with no periodic conditions in the $x$ direction. A second array was referred to by Shim and co-workers as the vertical close-packed array as shown in Fig. 11a. This packing has the representative periodic cell shown in Fig. 11b. Again, only the domain shown in Fig. 11b was modeled and the only periodic conditions 


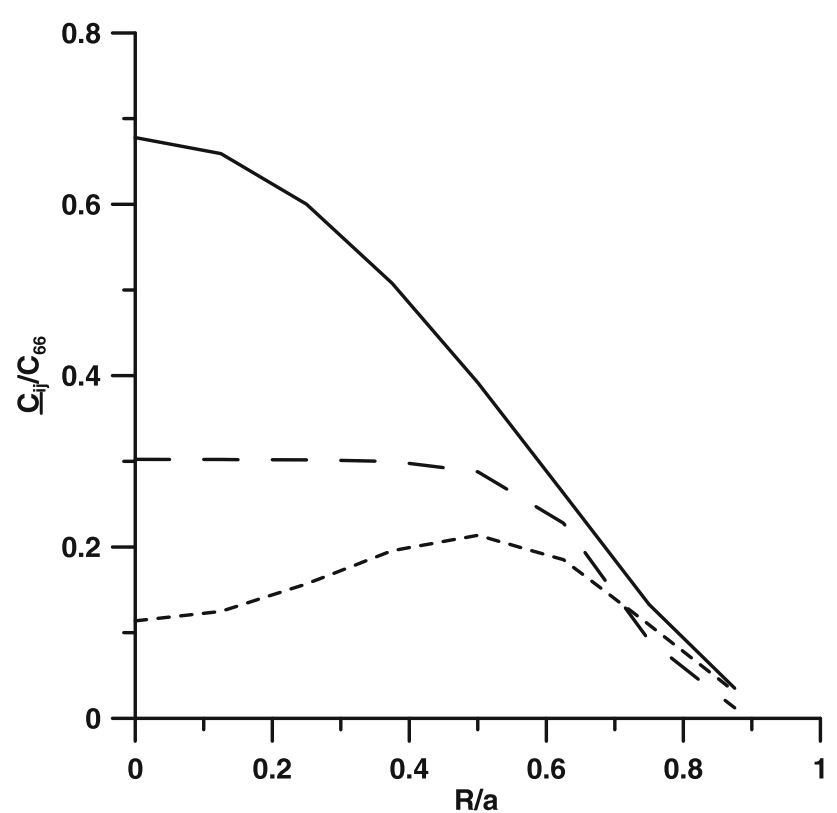

Fig. 8 The effective elastic constants for the hollow particles

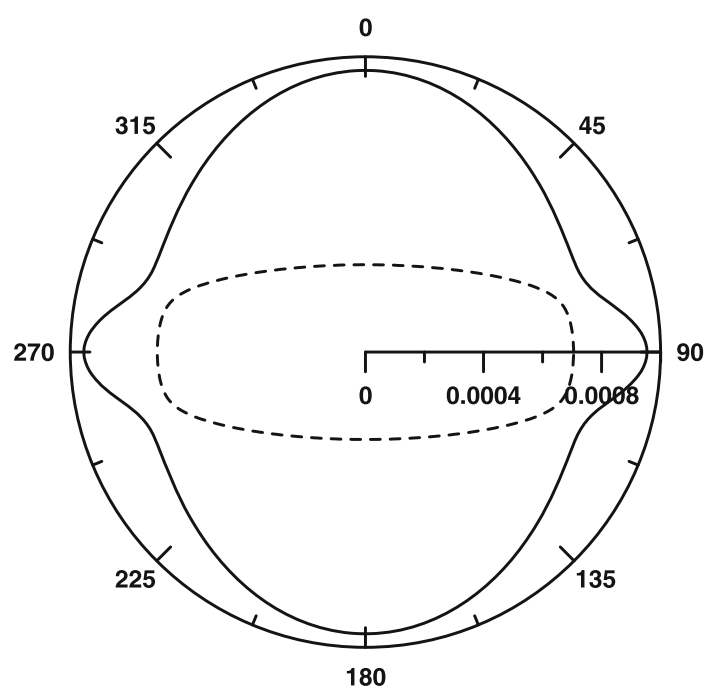

Fig. 9 Slowness curves for the orthotropic particles

that were imposed were those on the lines that border the lower and upper tangents of the periodic cell. In all meshes used for the analysis, four elements were used in the thickness direction of the ring with circumferential divisions every $10^{\circ}$.

In the experiments of Shim and co-workers, the wave speed was measured by initiating impact along the upper region of the packing by dropping a mass from various heights. There was some height-dependence of the wave speed for the various materials and packings used. In the present analysis, the wave vector was specified to be directed in the $y$ direction $(\theta=0)$ and the longitudinal wave speeds were computed in the large wavelength limit by taking the a
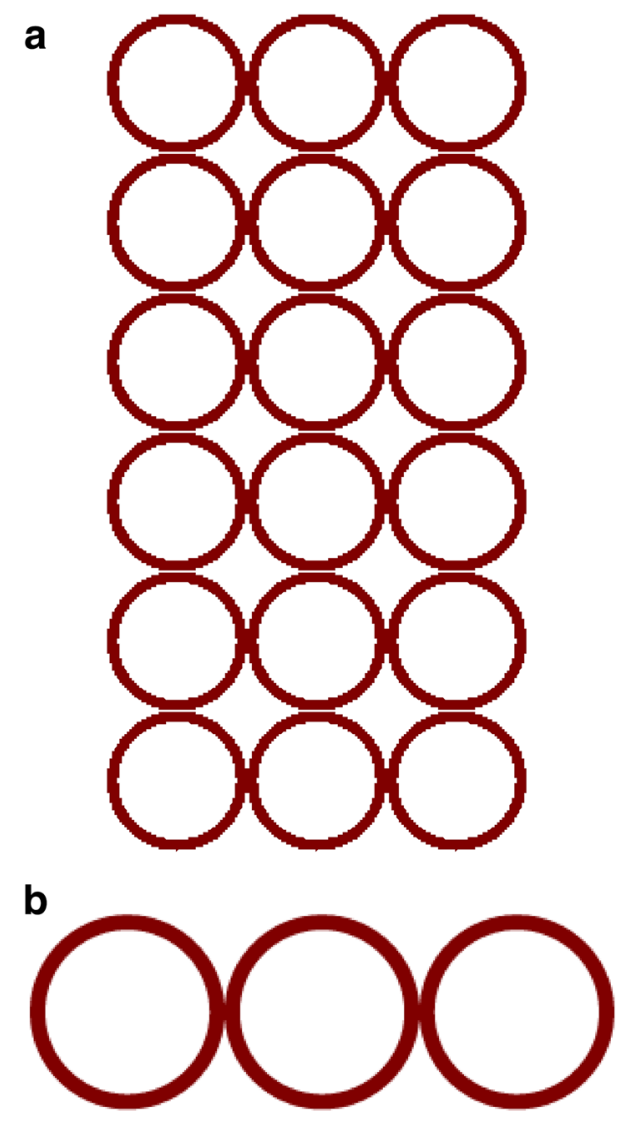

Fig. 10 a The square-packed experimental array of Shim and coworkers [31]. b The representative unit cell for the square-packed array

linear extension of the dispersion curve as was used for earlier analyses. There were a total of four packings used: a square packing of aluminum (packing 1), a square packing of brass (packing 2), a vertical close-packed of brass (packing 3), and a vertical close-packed of aluminum (packing 4).

The results of this analysis and comparison are shown in Fig. 12, and are shown as solid points as computed by the numerical model compared with the range of experimental wave speeds as denoted by a solid line. The agreement is reasonably good, especially considering the small number of rows of rings used in the $y$ direction in the experiments, and demonstrates at least in part the accuracy that can be expected for earlier wave speed results.

\section{Conclusions}

The kinematic assumptions imposed in this study are significant, and represent a limiting constraint on the actual behavior of particle interactions. Within the context of those assumptions, however, several broad conclusions can be stated: 


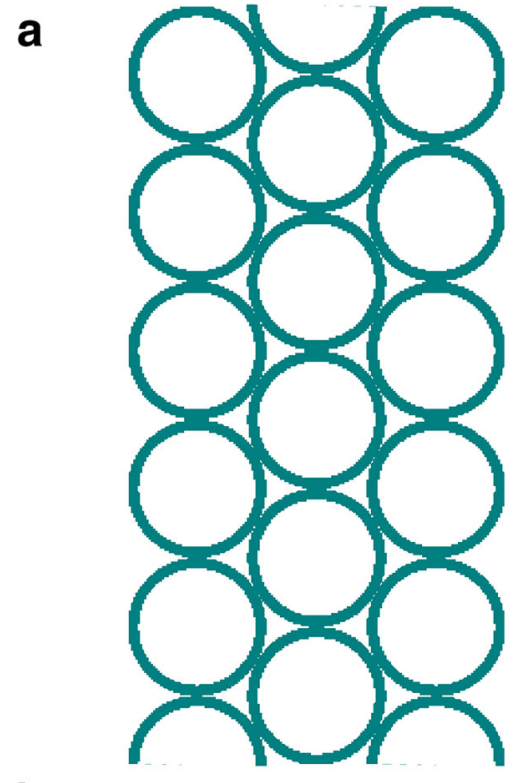

b

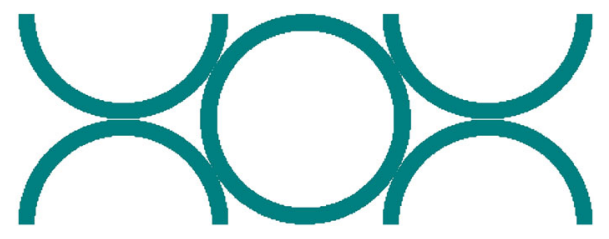

Fig. 11 a The vertical close-packed experimental array of Shim and co-workers [31]. b The representative unit cell for the vertical closepacked array

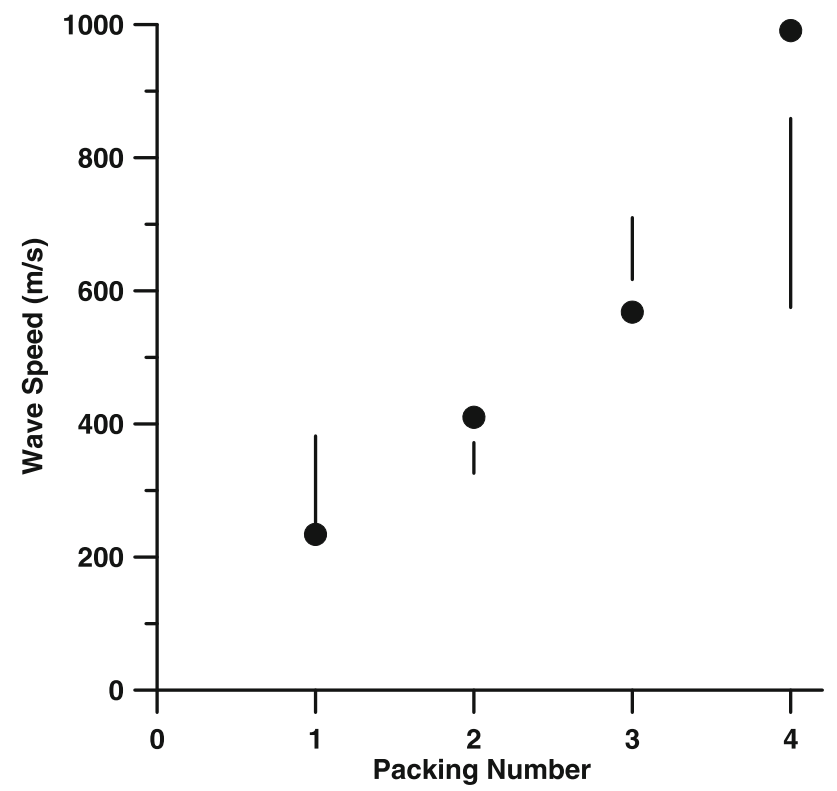

Fig. 12 Comparison of predicted (solid dots) versus experimentally measured (solid lines) wave speeds

1. The basic disperson relations of both solid and hollow circular discs under square packing follow the same general trend as continuous media with a shift downward in frequency of at least $50 \%$. The lowest shear and longitudinal modes maintain their position in the frequency spectrum.

2. Fully solid particles maintain elastic behavior that is essentially isotropic, but this behavior changes as the particle transitions into a thin ring.

3. The effective cubic elastic constants have widely varying trends as the particle becomes hollow, with relative reductions in magnitude reducing their value to roughly one-fourth of their particle value.

4. In comparisons with experiment, the longitudinal wave speeds predicted by the present approach agree reasonably well with experimental wave speeds determined from small planar packings of square-packed and vertical close-packed thin ring assemblies.

\section{References}

1. Duffy J, Mindlin RD (1957) Stress-strain relations and vibrations of a granular medium. ASME J Appl Mech 24:585-593

2. Deresiewicz H (1958) Mechanics of granular media. Adv Appl Mech 5:233-306

3. Deresiewicz H (1958) Stress-strain relation for a simple model of a granular medium. ASME J Appl Mech 25:402-406

4. Digby PJ (1981) The effective elastic modulus of porous granular rocks. ASME J Appl Mech 48:803-808

5. Walton K (1987) The effective elastic moduli of a random packing of spheres. J Mech Phys Solids 35:213-226

6. Goddard JD (1990) Nonlinear elasticity and pressure-dependent wave speeds in granular media. Proc R Soc Lond 430:105-131

7. Velicky B, Caroli C (2002) Pressure dependence of the sound velocity in a two-dimensional lattice of Hertz-Mindlin balls: mean-field description. Phys Rev E 65:021307

8. Coste C, Gilles B (1999) On the validity of Hertz contact law for granular material acoustics. Eur Phys J B 7:155-168

9. Melin S (1994) Wave propagation in granular assemblies. Phys Rev E 49:2353-2368

10. Jia X, Caroli C, Velicky B (1999) Ultrasound propagation in externally stressed granular media. Phys Rev Lett 82(8):1863-1866

11. Lanier J, Jean M (2000) Experiments and numerical simulations with 2D disks assembly. Power Technol 109:206-221

12. Hostler SR, Brennan CE (2005) Pressure wave propagation in a shaken granular bed. Phys Rev E 72:031303

13. Somfai E, Roux J-N, Snoeijer JH, van Hecke M, van Saarloos W (2005) Elastic wave propagation in confined granular systems. Phys Rev E 72:021301

14. Mouraille O, Mulder WA, Luding S (2006) Sound wave acceleration in granular materials. J Stat Mech: Theory Exp 7:P07023

15. Mouraille O, Herbst O, Luding S (2009) Sound propagation in isotropically and uni-axially compressed cohesive, frictional granular solids. Eng Fract Mech 76:781-792

16. Henann DL, Valenza JJ, Johnson DL, Kamrin K (2013) Smallamplitude acoustics in bulk granular media. Phys Rev E 88:042205

17. Digby PJ (1981) The effective elastic moduli of porous granular rock. ASME J Appl Mech 48:803-808

18. Dvorkin J, Nur A, Yin H (1994) Effective properties of cemented granular material. Mech Mater 18:351-366

19. Langlois V, Jia X (2011) Ultrasonic monitoring of the elastic properties of PMMA bead packings and their rearrangement during pressure sintering. Powder Technol 208:509-514 
20. Langlois V, Jia X (2014) Acoustic probing of elastic behavior and damage in weakly cemented granular material. Phys Rev E 89:023206

21. Yu CW, Thompson EG, Heyliger PR (2004) The compaction of aggregates of non-spherical linear viscous particles. Comput Methods Appl Mech Eng 192:4929-4946

22. Cundall PA, Strack ODL (1979) A discrete numerical model for granular assemblies. Geotechnique 1:47-65

23. Heyliger PR, McMeeking RM (2001) Cold plastic compaction of powders by a network model. J Mech Phys Solids 49:2031-2054

24. Redanz P, Fleck NA (2001) The compaction of a random distribution of metal cylinders by the discrete element method. Acta Mater 49:4325-4335

25. Reddy JN (1993) An introduction to the finite element method. McGraw-Hill, New York

26. Auld BA (1990) Acoustic fields and waves in solids, 2nd edn. Krieger Publishing Company, Malabar
27. Hladky-Hennion AC, Decarpigny JN (1991) Analysis of the scattering of a plane acoustic wave by a doubly periodic structure using the finite element method: Application to Alberich anechoic coatings. J Acoust Soc Am 90:3356-3367

28. Langlet P, Hladky-Hennion AC, Decarpigny JN (1995) Analysis of the propagation of plate acoustic waves in passive periodic materials using the finite element method. J Acoust Soc Am 98:2792-2800

29. Hyer MW (1998) Stress Anal Fiber-Reinf Comp Mater. McGrawHill, Boston

30. Nayfeh AH (1995) Wave Propag Layer Anisotropic Media. Elsevier, Amsterdam

31. Shim VPW, Lan R, Guo YB, Yang LM (2007) Elastic wave propagation in cellular systems - experiments on single rings and ring systems. Int J Impact Eng 34:1565-1584 